\title{
Biochemical Properties of Akazara Scallop Myosin Hybridized with the Foreign Regulatory Light Chains
}

\author{
Takao Ojima* and Kiyoyoshi Nishita* \\ (Received June 9, 1989)
}

\begin{abstract}
The roles of regulatory light chains in the biochemical function of molluscan myosin were studied by hybridizing "desensitized" akazara scallop myosin with the regulatory light chains of rabbit skeletal, chicken gizzard, rainbow trout dorsal, and crayfish abdominal myosins. By measuring the Mg-ATPase activities of myosin and actomyosin, and the superprecipitation activity of actomyosin, the following results were obtained:

1) All the foreign regulatory light chains we tested, led to the inhibition of Mg-ATPase activities of both desensitized myosin and acto-desensitized myosin in the absence of $\mathrm{Ca}^{2+}$. Whereas, whether the inhibition of $\mathrm{Mg}$-ATPase activities was neutralized by $\mathrm{Ca}^{2+}$ or not, i.e. $\mathrm{Ca}^{2+}$-sensitivity of the Mg-ATPase activities recovered or not, was dependent on the species of the regulatory light chain. In this respect, these regulatory light chains could be classified into two groups; One includes the regulatory light chains of akazara scallop, chicken gizzard, and crayfish abdominal myosins, which afford the $\mathrm{Ca}^{2+}$-sensitivity to desensitized myosin, and the other includes those of rabbit skeletal and rainbow trout dorsal myosins, which afford no $\mathrm{Ca}^{2+}$-sensitivity.

2) The superprecipitation activity of skeletal acto-akazara scallop myosin was remarkably decreased by the removal of regulatory light chains. However, the decreased superprecipitation activity recovered on the binding of either akazara scallop regulatory light chains or foreign regulatory light chains. These results may indicate that foreign regulatory light chains as well as molluscan regulatory light chains can maintain the myosin conformation that is indispensable for actin-myosin interaction.
\end{abstract}

Since 1970, Kendrick-Jones, Szent-Györgyi, and their associates ${ }^{1-7}$ have demonstrated that $\mathrm{Ca}^{2+}$-regulatory protein in molluscan muscles is the specific light chain subunits of myosin called "EDTA-light chain" or "regulatory light chain". Based mainly on their findings on desensitization and resensitization experiments of myofibrils, and on $\mathrm{Ca}^{2+}$-sensitivity measurements of heavy meromyosin and subfragment1 , they proposed that the role of regulatory light chains in $\mathrm{Ca}^{2+}$-regulation is to inhibit the interaction between actin and myosin uy blocking the sites on myosin instead of the sites on actin in the case of vertebrate muscles, and postulated that cooperation between two moles of regulatory light chains or two moles of myosin heads is necessary for $\mathrm{Ca}^{2+}$-regulation. ${ }^{5-7)}$

However, we could not observe such a cooperativity in the desensitization and resensitization of adductor muscle myosin of akazara scallop Chlamys nipponensis akazara. ${ }^{8)}$ Similar results were obtained with ezo-giant scallop patinopecten yessoensis adductor muscle myo$\sin ^{\theta)}$ and clam Meretrix lusoria foot muscle myosin. ${ }^{10)}$ Moreover, Asada et al, ${ }^{11)}$ and Ashiba et $a l,{ }^{10)}$ found that the Mg-ATPase activity of clam foot myosin alone is as sensitive as that of skeletal acto-clam foot myosin, and that the desensitization of clam foot myosin is accompanied by the reversible loss of superprecipitation activity of the actomyosin. From these findings, they proposed that the primary role of the regulatory light chain in $\mathrm{Ca}^{2+}$-regulation is to inhibit myosin ATPase activity not actin-myosin interaction, and the secondary role is to maintain the conformation of myosin heads which is essential for actin-myosin interaction. ${ }^{11}$ Thereafter, these findings were confirmed with myosins from squid Todarodes pacificus, ${ }^{12)}$ ezo-giant scallop, ${ }^{12}$ ) akazara scallop, ${ }^{18-17)}$ and surf-clam Spisula sacharinen$s i s,{ }^{18)}$ and extended to the tension development

* Laboratory of Biopolymer Chemistry, Faculty of Fisheries, Hokkaido University, Hakodate 041, Japan (尾岛孝男，西田清義：北海道大学水産学部水産高分子化学諈座).

Abbreviations: EGTA, ethyleneglycol-bis( $\beta$-aminoethylether)-N, $\mathbf{N}^{\prime}$-tetraacetate; DTT, dithiothreitol. ITP, inosine-5'-triphosphate. 
of glycerinated muscle fibers of ezo-giant scallop. ${ }^{19)}$ Moreover, it was directly observed that the myosin heads of bay-scallop Aequipecten irradians were shrunk by the removal of the regulatory light chains under electronmicroscope observation. ${ }^{20)}$

In 1980 , Sellers et al. $^{21)}$ found that desensitized bay-scallop myofibrils could be hybridized with more than ten species of foreign regulatory light chains, and examined the actinactivated $\mathrm{Mg}$-ATPase activity and $\mathrm{Ca}^{2+}$ binding property of hybridized myofibrils. As a result, these foreign regulatory light chains were found to be classified into three major groups with respect to $\mathrm{Ca}^{2+}$-sensitizing ability; i.e. a) the regulatory light chains which afford high sensitivity and high affinity to $\mathrm{Ca}^{2+}$ on desensitized myofibrils (molluscan regulatory light chains), b) the regulatory light chains which afford low sensitivity and low affinity to $\mathrm{Ca}^{2+}$ on desensitized myofibrils (chicken gizzard and insect regulatory light chains), and c) the regulatory light chains which afford no sensitivity and no affinity to $\mathrm{Ca}^{2+}$ on desensitized myofibrils (skeletal and cardiac regulatory light chains). On the other hand, we have attempted to determine the biochemical properties of regulatory light chains by using hybridized akazara scallop myosin with the foreign regulatory light chains of vertebrate skeletal and smooth muscle myosins. ${ }^{22-24)}$

In the present paper, we examine the effects of hybridization of foreign regulatory light chains from rabbit skeletal, rainbow trout dorsal, chicken gizzard, and crayfish abdominal myosins on the biochemical properties of akazara scallop myosin, and show that all regulatory light chains can inhibit myosin $\mathrm{Mg}$ ATPase activity and restore the superprecipitation ability of acto-desensitized myosin. In addition, we could classify these regulatory light chains into two groups with respect to $\mathrm{Ca}^{2+}$-sensitizing ability.

\section{Materials and Methods}

Akazara scallop myosin was prepared from striated adductor muscles by the method of Nishita et al. ${ }^{8)}$ Desensitization of myosin was performed by EDTA-washing at $=0-4^{\circ} \mathrm{C}{ }^{\text {s) }}$ Rabbit skeletal and chicken gizzard myosins were prepared by the methods of Perry, ${ }^{26)}$ and Ikebe et al., ${ }^{26)}$ respectively. Crayfish abdomi- nal and rainbow trout dorsal myosins were prepared by the same method as for akazara scallop myosin. ${ }^{8)}$ The regulatory light chains of these myosins were prepared by the ureaacetone precipitation method described in our previous paper. ${ }^{28)}$ Rabbit actin was prepared according to the method of Spudich and Watt. ${ }^{27)}$ Either the resensitized myosin or hybridized myosin was prepared as follows: Desensitized akazara scallop myosin was mixed with the respective regulatory light chains in $0.6 \mathrm{M} \mathrm{KCl}, 4 \mathrm{~mm} \mathrm{MgCl}_{2}, 50 \mathrm{~mm}$ Tris maleate (pH 7.0) and $0.5 \mathrm{~mm}$ DTT, left standing at $4^{\circ} \mathrm{C}$ for $4 \mathrm{~h}$, and diluted with $10 \mathrm{vol}$. of cold water. The precipitates thus formed, were collected by centrifugation at $10,000 \times g$ for 5 min, removing the excess and unbound regulatory light chains into the supernatant, dissolved in and dialyzed against $0.6 \mathrm{M} \mathrm{KCl}, 50$ $\mathrm{mm}$ Tris maleate ( $\mathrm{pH} 7.0$ ), and $0.5 \mathrm{~mm}$ DTT. Mg-ATPase and superprecipitation activities were measured under the same conditions as described in a previous report. ${ }^{14)}$ Inorganic phosphate liberated by ATP hydrolysis was determined by the method of Youngburg and Youngburg. ${ }^{28)}$ Free $\mathrm{Ca}^{2+}$ concentration in the reaction mixture was varied with the Ca-EGTA buffer adopting the stability constant of CaEGTA chelate as $8.45 \times 10^{5} \mathrm{M}^{-1}{ }^{29}$ ) When ITPinduced superprecipitation was determined, the reaction was initiated by the addition of $1 \mathrm{~mm}$ ITP instead of $1 \mathrm{mM}$ ATP. SDS- and ureapolyacrylamide gel electrophoresis were carried out according to the methods of Weber and Osborn, ${ }^{30)}$ and Perrie and Perry, ${ }^{31}$ ? respectively. Gel rods were stained and destained as described previously. ${ }^{15)}$ The amounts of regulatory light chains bound to myosin were determined by scanning the urea-gel rods using the Shimadzu CS-9000 Chromatoscanner. The staining intensity was corrected by using the staining ratio of regulatory light chain to SHlight chain as 1.2 (regulatory light chain/SHlight chain). Up to $2 \mathrm{~mol}$ of the foreign regulatory light chains were shown to bind to $1 \mathrm{~mol}$ of desensitized akazara scallop myosin. The protein concentration was determined by the biuret method. ${ }^{\text {s2) }}$

\section{Results and Discussion}

Effects of Foreign Regulatory Light Chains on Mg-ATPase Activities of Both Akazara Scallop 


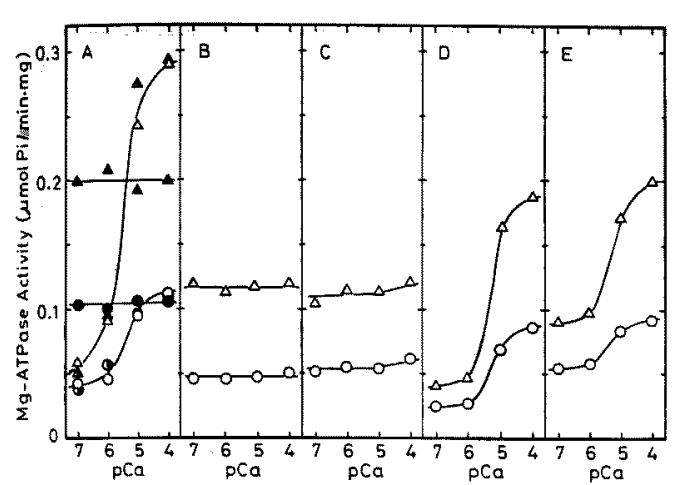

Fig. 1. Effects of foreign regulatory light chains on the Mg-ATPase activities of both akazara scallop desensitized myosin and acto-desensitized myosin. ATPase activity was measured at $15^{\circ} \mathrm{C}$ in a medium containing $30 \mathrm{mM} \mathrm{KCl}, 20 \mathrm{~mm}$ Tris maleate ( $\mathrm{pH} 6.8), 2 \mathrm{~mm} \mathrm{MgCl}_{2}, 1 \mathrm{~mm}$ ATP, $0.1 \mathrm{mg} / \mathrm{m} l$ myosin $(O, \bullet, 0)$ or $0.1 \mathrm{mg} / \mathrm{ml}$ myosin plus $0.05 \mathrm{mg} / \mathrm{m} l \mathrm{~F}$-actin $(\triangle, \Delta, \Delta)$, and $0.1 \mathrm{~mm}$ EGTA plus various concentrations of $\mathrm{CaCl}_{2}$. Free $\mathrm{Ca}^{2+}$ concentration was calculated using a stability constant of $8.45 \times 10^{5} \mathrm{M}^{-1}$ for $\mathrm{Ca}$ EGTA chelate, ${ }^{2 \theta}$ and expressed in terms of $\mathrm{pCa}$ $\left(=-\log \left[\mathrm{Ca}^{2+}(\mathrm{M})\right]\right)$. A) $O, \Delta$, untreated myosin and actomyosin;,$\Delta$, desensitized myosin and actomyosin; $\mathbf{\Lambda}, \boldsymbol{\Delta}$, resensitized myosin and actomyosin. B) $\sim$ E), hybridized myosins with rabbit, rainbow trout, chicken gizzard, and crayfish regulatory light chains, respectively. Amounts of bound foreign regulatory light chains were estimated to be about 2 mol per 1 mol of desensitized myosin by urea-gel electrophoresis and densitometry.

\section{Desensitized Myosin and Acto-desensitized Myosin}

Fig. 1A shows the Mg-ATPase activities of both akazara scallop myosin alone and skeletal acto-akazara scallop myosin measured as a function of $\mathrm{Ca}^{2+}$ concentration. The activities of myosin alone and actomyosin were both increased by the same concentration of $\mathrm{Ca}^{2+}$; the largest activation was observed between $10^{-8} \sim 10^{-5} \mathrm{M}$. On the other hand, complete removal of the regulatory light chains from myosin by EDTA-washing ${ }^{8}$ ) caused an increase in the myosin Mg-ATPase activities at low concentrations of $\mathrm{Ca}^{2+}$, decreasing the $\mathrm{Ca}^{2+}$ sensitivity. Desensitization of actomyosin $\mathrm{Mg}$ ATPase, however, resulted not only from an increase in the activities at low concentrations of $\mathrm{Ca}^{2+}$ but also from a decrease in those at high concentrations of $\mathrm{Ca}^{2+}$. The decrease in actomyosin ATPase activities at high concentrations of $\mathrm{Ca}^{2+}$ by the removal of regulatory light chains seems to have resulted from the subsequent conformational change of myosin heads as suggested by Asada et al. ${ }^{11)}$ and Suzuki et al. ${ }^{18)}$ The recombination of akazara scallop regulatory light chains to desensitized myosin brought about a full resensitization of the ATPase activities of both myosin and actomyosin. On the other hand, the binding of the rabbit skeletal DTNB-light chain led to a decrease in ATPase activities of both myosin and actomyosin, recovering no $\mathrm{Ca}^{2+}$-sensitivity (Fig. 1B). Rainbow trout regulatory light chains also could not confer the $\mathrm{Ca}^{2+}$-sensitivity (Fig. 1C). Essentially the same results have already been reported regarding hybridized myosins with Alaska pollack and Pacific pomfret regulatory light chains. ${ }^{s 3)}$ Thus, fish regulatory light chains appeared unable to confer the $\mathrm{Ca}^{2+}$ sensitivity to desensitized myosin like rabbit regulatory light chains. ${ }^{22-24)}$ On the other hand, both the chicken gizzard and the crayfish regulatory light chains could confer the $\mathrm{Ca}^{2+}$ sensitivity to the desensitized myosin (Fig.1 $D, E$ ), although the extent of maximal activation was somewhat lower than that of untreated myosin. Yet, half activation of the Mg-ATPase activities of these hybridized myosins was observed at around $3 \times 10^{-\theta} \mathrm{M}$, indicating that the affinity of akazara scallop myosin to $\mathrm{Ca}^{2+}$ is not changed so greatly by the replacement of the regulatory light chains with chicken gizzard or crayfish regulatory light chains. This agreed well with our previous report, ${ }^{242}$ in which we showed that the high affinity specific $\mathrm{Ca}^{2+}$ binding sites were recovered not only by the recombination of akazara scallop regulatory light chains but also by hybridization of chicken gizzard regulatory light chains. In contrast, Sellers et al. ${ }^{21)}$ reported that the replacement of the regulatory light chains of scallop myofibrils by the chicken gizzard or insect regulatory light chains brought about the decrease in the affinity to $\mathrm{Ca}^{2+}$. The reason for this inconsistency between our results and Sellers' remains obscure, but technically, the determination of the $\mathrm{Ca}^{2+}$ binding seems to be more difficult and complicated with myofibrils than myosin because myofibrils contain other $\mathrm{Ca}^{2+}$ binding protein such as troponin ${ }^{84,86)}$ in addition to myosin. 

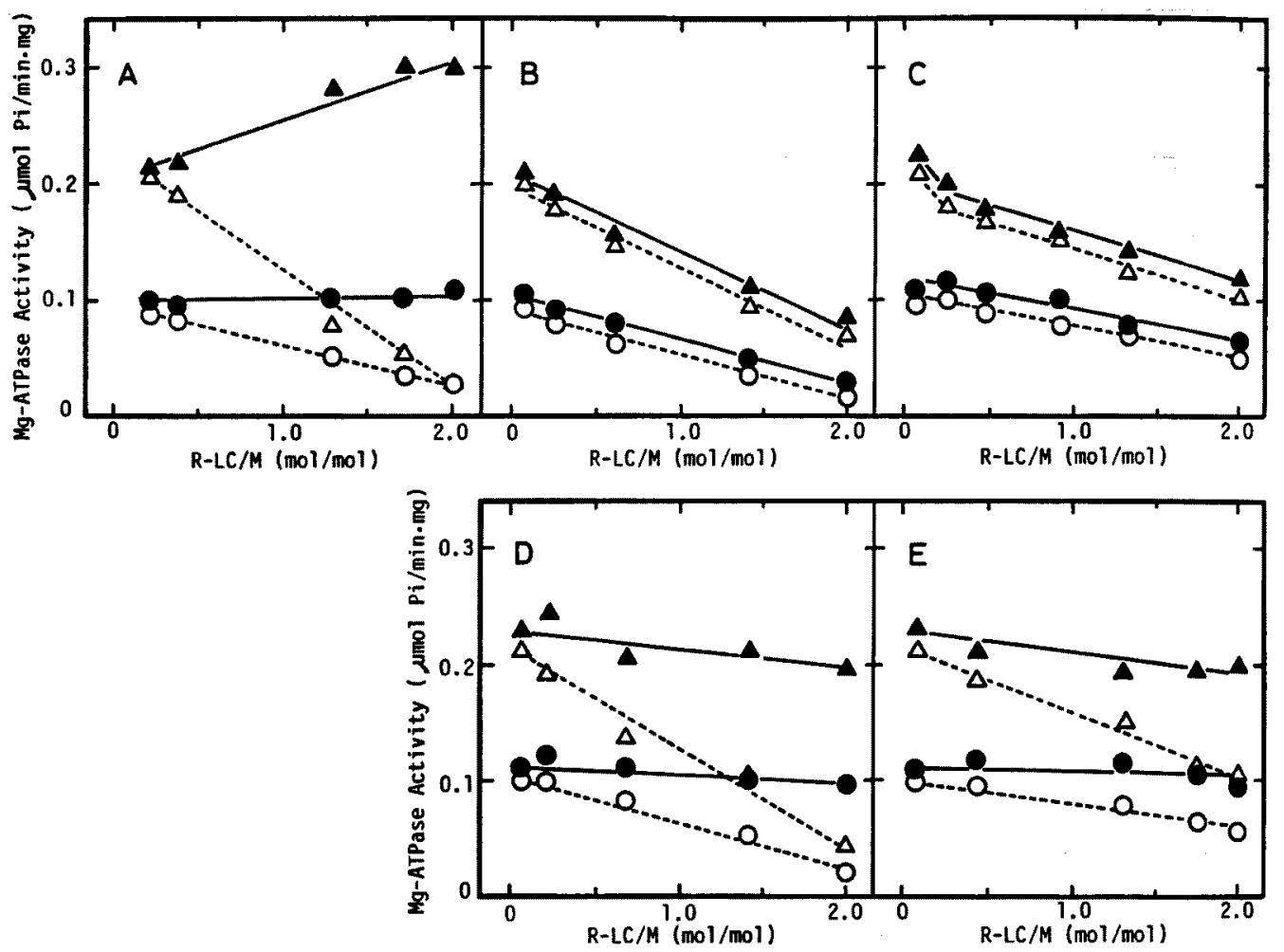

Fig. 2. Relationship between the Mg-ATPase activity and the amount of bound regulatory light chains. Desensitized akazara scallop myosin was recombined or hybridized with various amounts of the regulatory light chains of akazara scallop (A), rabbit (B), rainbow trout $(C)$, chicken gizzard (D), and crayfish (E). The regulatory light chain content was estimated by urea-gel electrophoresis and densitometry, and is shown on the abscissa. The Mg-ATPase activities of myosin $(O,-)$ and actomyosin $(\Delta, \Delta)$ were measured in the presence $(\bullet, \Delta)$ or absence $(O, \Delta)$ of $0.1 \mathrm{~mm} \mathrm{Ca}{ }^{2+}$. Other conditions were the same as given in Fig. 1. R-LC, regulatory light chain; M, myosin.

Relationship between Mg-ATPase Activity and Amount of Bound Regulatory Light Chains

In the binding of akazara scallop regulatory light chains to desensitized myosin, the $\mathrm{Mg}$ ATPase activities of both myosin and actomyosin were decreased linearly with an increase in the amount of the regulatory light chain in the absence of $\mathrm{Ca}^{2+}$ (Fig. 2A). However, in the presence of $\mathrm{Ca}^{2+}$, the activity of myosin alone was practically unchanged, but that of actomyosin was increased linearly with an increase in the amount of the regulatory light chain. This may correspond to the recovery of myosin conformation which is essential for normal actin-myosin interaction. On the other hand, when rabbit regulatory light chains were hybridized, the activity of actomyosin as well as that of myosin alone was reduced irrespective of the presence or absence of $\mathrm{Ca}^{2+}$ (Fig. 2B). Essentially the same results were obtained in the case of rainbow trout regulatory light chains (Fig. 2C). Whereas, upon recombining with the gizzard regulatory light chains, the Mg-ATPase activities of both myosin and actomyosin were inhibited in the absence of $\mathrm{Ca}^{2+}$ to almost the same extent as in the case of akazara scallop and rabbit regulatory light chains, and was rather slightly inhibited even in the presence of $\mathrm{Ca}^{2+}$ unlike in the case of the akazara scallop regulatory light chain (Fig. 2D). The slight inhibition in the presence of $\mathrm{Ca}^{2+}$ had been reported to be diminished by phosphorylation of the regulatory light chains. ${ }^{22)}$ As shown in Fig. 3E, the crayfish regulatory light chains restored the $\mathrm{Ca}^{2+}$-sensitivity of both desensitized myosin 

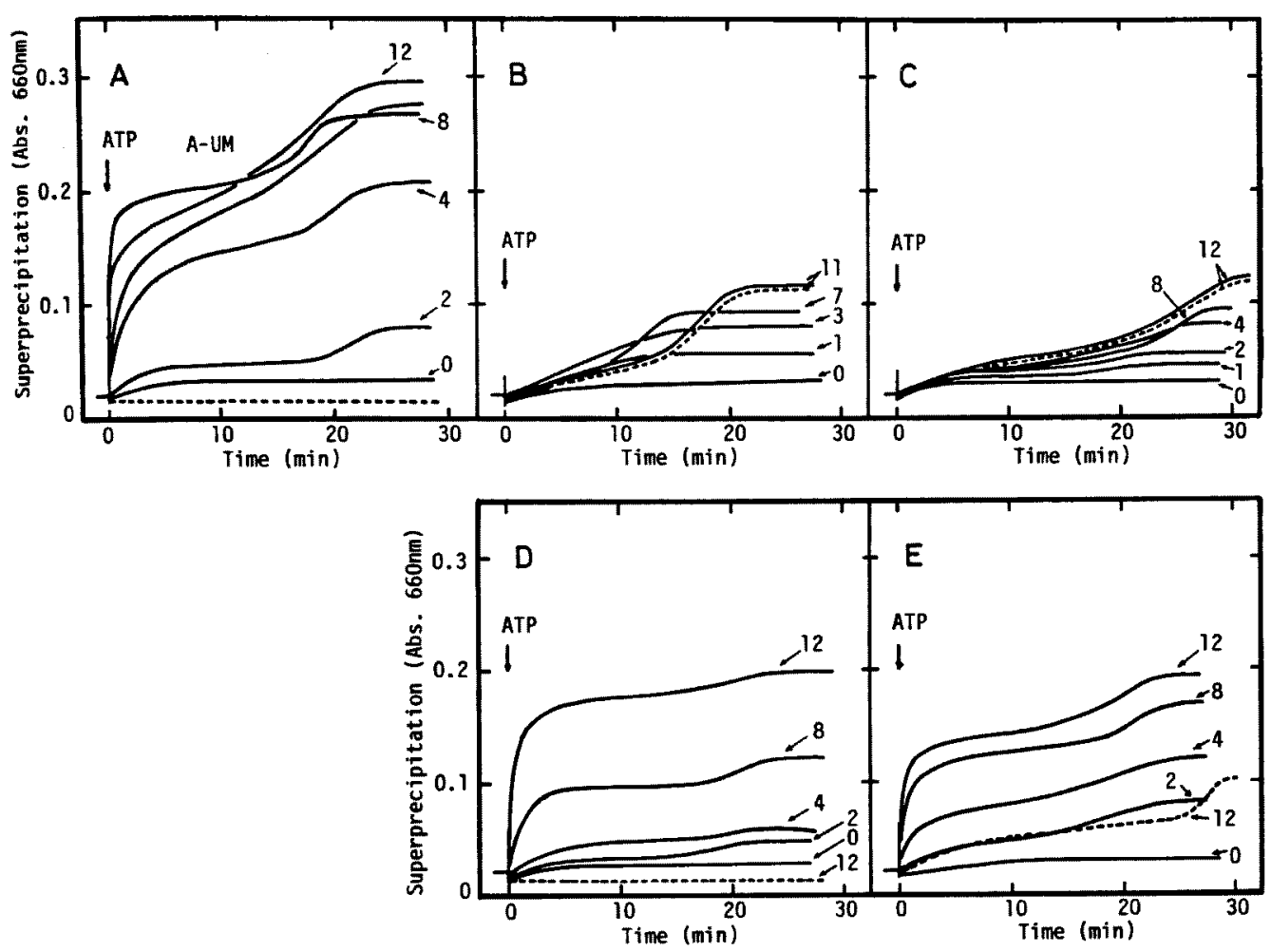

Fig. 3. Recovery of the superprecipitation activity of acto-desensitized myosin on binding of the foreign regulatory light chains. Desensitized akazara scallop myosin was recombined with the regulatory light chains (A) and hybridized with the regulatory light chains of rabbit (B), rainbow trout (C), chicken gizzard (D), and crayfish (E), respectively. Superprecipitation reaction was carried out under the same conditions for Mg-ATPase assay. - and -- , in the presence and absence of $0.1 \mathrm{mM} \mathrm{Ca}{ }^{2+}$, respectively. Numerical figures with arrow marks attached to superprecipitation curves are the values for the amount of the regulatory light chain added to desensitized myosin (weight percent to the desensitized myosin). A-UM, acto-untreated myosin.

and acto-desensitized myosin in a similar manner as did the gizzard regulatory light chains. However, the extent of $\mathrm{Ca}^{2+}$-sensitivity conferred by the crayfish regulatory light chains was considerably lower than that by the akazara scallop or chicken gizzard regulatory light chains. One possible explanation for the low $\mathrm{Ca}^{2+}$-sensitivity of this hybridized myosin is the unsuitable ATPase-assay condition, since myosin-linked regulation in crayfish muscle was reported to be strongly dependent on the ATPase-assay conditions; e.g. $\mathrm{Ca}^{2+}$-sensitivity was only observed at $\mathrm{NaCl}$ concentration greater than $86 \mathrm{~mm}^{86}$ )

From these results, it became evident that all regulatory light chains examined so far can inhibit the Mg-ATPase activity of desensitized akazara scallop myosin. Accordingly, it is confirmed that the primary role of regulatory light chains is to inhibit myosin ATPase as proposed by Asada et al. ${ }^{11}$ Moreover, these regulatory light chains could be classified into two groups with respect to their $\mathrm{Ca}^{2+}$-sensitizing ability: One is the $\mathrm{Ca}^{2+}$-sensitive group which is capable of restoring $\mathrm{Ca}^{2+}$-sensitivity to desensitized myosin (akazara scallop, chicken gizzard, and crayfish regulatory light chains), and the other is $\mathrm{Ca}^{2+}$-insensitive group which is incapable of restoring $\mathrm{Ca}^{2+}$-sensitivity to desensitized myosin (rabbit and rainbow trout regulatory light chains). Sellers et al. ${ }^{21}$ ) had reported that the regulatory light chains can be classified into three groups with respect to the $\mathrm{Ca}^{2+}$-sensitizing ability. Our classification is 


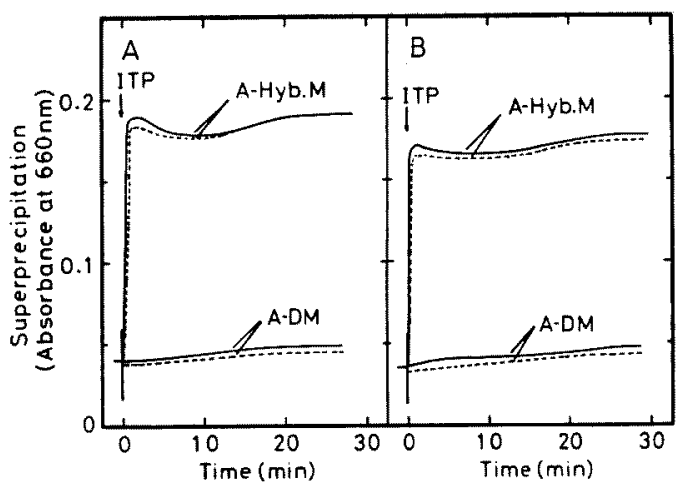

Fig. 4. ITP-induced superprecipitation of actohybridized myosins with rabbit and rainbow trout regulatory light chains. Desensitized myosin was hybridized with the regulatory light chains of rabbit (A) and rainbow trout (B), respectively. The amount of bound regulatory light chains of either hybrid myosin was estimated to be about $2 \mathrm{~mol}$ per mol myosin by urea-gel electrophoresis. The superprecipitation of acto-hybridized myosin was induced by the addition of $1 \mathrm{~mm}$ ITP instead of $1 \mathrm{~mm}$ ATP. Other conditions were the same as in Fig. 3. - and ---, in the presence and absence of $0.1 \mathrm{mM} \mathrm{Ca}^{2+}$, respectively. A-DM, actodesensitized myosin; A-Hyb. M, acto-hybridized myosin.

qualitatively consistent with that of Sellers et al., ${ }^{21}$ but we did not subdivide the $\mathrm{Ca}^{2+}$-sensitive light chains into high- and lowsensitive groups since the extent of the $\mathrm{Ca}^{2+}$ sensitivity and the affinity of hybrid myosin to $\mathrm{Ca}^{2+}$ appeared to vary depending on the assay conditions.

Here, we should mention the cooperative restoration theory of $\mathrm{Ca}^{2+}$-sensitivity accompanied by the recombination of regulatory light chains. Chantler and Szent-Györgyi ${ }^{7)}$ showed that the Mg-ATPase activity of desensitized scallop myofibrils in the absence of $\mathrm{Ca}^{2+}$ remains constant until one mole of regulatory light chain is added back, then is depressed linearly upon further re-addition of regulatory light chains. Moreover, they postulated that this ATPase dependency is taken as evidence that two moles of regulatory light chain must be attached to one mole of intact scallop myosin for regulation, and that the recombination of the regulatory light chains occurs in a negatively cooperative manner. However, we could not recognize such a cooperativity in preparing resensitized myosin and any hybrid myosins; i.e. the activities in the absence of $\mathrm{Ca}^{2+}$ were linearly inhibited by increasing amounts of regulatory light chains. (see Fig. $2 A \sim E)$. The linear inhibition of the ATPase activity of desensitized myosin had also been reported with ezogiant scallop striated adductor myosin, $\left.{ }^{9}\right)$ surfclam foot myosin, ${ }^{18}$ akazara scallop smooth adductor myosin, ${ }^{18}$ and akazara scallop striated adductor myofibrils. ${ }^{87)}$ The reason for this discrepancy is still under investigation.

Effects of Regulatory Light Chain on the Super-Precipitation of Skeletal Acto-desensitized Akazara Scallop Myosin

As shown in Fig. 3A, the superprecipitation activity of acto-untreated myosin was high in the presence of $\mathrm{Ca}^{2+}$, but that of acto-desensitized myosin was remarkably reduced. The reduced superprecipitation activity of acto-desensitized myosin was, however, fully recovered by the recombination of akazara scallop regulatory light chains. When rabbit regulatory light chains were hybridized, the reduced superprecipitation activity of acto-desensitized myosin was also recovered, although no $\mathrm{Ca}^{2+}$ sensitivity was conferred even with the full recombination of the regulatory light chains (Fig. 3B). In addition, both the extent and the rate of superprecipitation were found to be very low as compared with those of acto-akazara scallop resensitized myosin. Rainbow trout regulatory light chains were also capable of restoring, though only slightly, the superprecipitation activity of acto-desensitized myosin (Fig. 3C). Therefore, these vertebrate skeletal regulatory light chains seem to be less effective in recovering superprecipitation ability. However, when superprecipitation was induced by ITP, the restoration was more clearly observed than by ATP. As shown in Fig. 4A and $B$, rabbit and rainbow trout regulatory light chains strongly restored superprecipitation activity. The reason for the high superprecipitation activity with ITP may be partly because of the approximately 5 times greater ITPase activity than ATPase activity (data not shown). On the other hand, the chicken gizzard regulatory light chains could recover either the superprecipitation activity of acto-desensitized myosin or its $\mathrm{Ca}^{2+}$-sensitivity, although the extent was slightly lower than that of acto-akazara 
scallop resensitized myosin. This agrees with the lower actin-activation of the Mg-ATPase activity (see Figs. 1 and 2). Crayfish regulatory light chains in the same way recovered superprecipitation activity and $\mathrm{Ca}^{2+}$-sensitivity as gizzard regulatory light chains did (Fig. 3E).

Taking these results into consideration, it may be concluded that all the regulatory light chains we tested, can potentially recover myosin conformation which is essential for normal interaction between myosin and actin. In addition, the recovery of $\mathrm{Ca}^{2+}$-sensitivity of superprecipitation activity was also dependent on the species of the light chain sources. In this respect, the regulatory light chains were able to be classified into two groups as in the case of Mg-ATPase.

\section{References}

1) J. Kendrick-Jones, W. Lehman, and A. G. Szent-Györgyi: J. Mol. Biol., 54, 313-326 (1970).

2) A. G. Szent-Györgyi, E. M. Szentkiralyi, and J. Kendrick-Jones: J. Mol. Biol., 74, 179-203 (1973).

3) J. Kendrick-Jones: Nature, 249, 631-634 (1974).

4) W. Lehman and A. G. Szent-Györgyi: J. Gen. Physiol., 66, 1-30 (1975).

5) J. Kendrick-Jones, E. M. Szentkiralyi, and A. G. Szent-Györgyi: J. Mol. Biol., 104, 747775 (1976).

6) R. M. Simmons and A. G. Szent-Györgyi: Nature, 273, 62-64 (1978).

7) P. D. Chantler and A. G. Szent-Györgyi: $J$. Mol. Biol., 138, 473-492 (1980).

8) K. Nishita, T. Ojima, and S. Watanabe: $J$. Biochem., 86, 663-673 (1979).

9) K. Konno, K. Arai, and S. Watanabe: $J$. Biochem., 86, 1639-1650 (1979).

10) G. Ashiba, K. Haraki, and S. Watanabe: $J$. Biochem., 91, 1795-1804 (1982).

11) T. Asada, G. Ashiba, and S. Watanabe: $J$. Biochem., 85, 1543-1546 (1979).

12) K. Konno, K. Arai, M. Yoshida, and S. Watanabe: J. Biochem., 89, 581-589 (1981).

13) T. Ojima, K. Nishita, and S. Watanabe: $J$. Biochem., 89, 1333-1335 (1981).
14) T. Ojima, K. Nishita, and S. Watanabe: $J$. Biochem., 93, 607-613 (1983).

15) T. Ojima and K. Nishita: Nippon Suisan Gakkaishi, 49, 1257-1264 (1983).

16) T. Ojima, H. Ichikawa, and K. Nishita: Nippon Suisan Gakkaishi, 52, 839-845 (1986).

17) T. Ojima and K. Nishita: Nippon Suisan Gakkaishi, 53, 93-98 (1987).

18) S. Hikichi, T. Ojima, S. Kakudate, and $K$. Nishita: Nippon Suisan Gakkaishi, 49, 141-148 (1983).

19) H. Suzuki, K. Konno, K. Arai, and S. Watanabe: J. Biochem., 88, 909-911 (1980).

20) P. F. Flicker, T. Wallimann, and P. Vibert: J. Mol. Biol., 169, 723-741 (1983).

21) J. R. Sellers, P. D. Chantler, and A. G. SzentGyörgyi: J. Mol. Biol. 144, 224-245 (1980).

22) T. Ojima, K. Nishita, and S. Watanabe: $J$. Biochem., 94, 307-310 (1983).

23) T. Ojima, K. Nishita, and S. Watanabe: $J$. Biochem., 96, 1109-1115 (1984).

24) H. Kishimura, T. Ojima, and K. Nishita: Nippon Suisan Gakkaishi, 52, 1469-1472 (1986).

25) S. V. Perry: in "Methods in Enzymology" (ed. by S. P. Colowick and N. O. Kaplan), Vol. 2, Academic Press, New York, 1955.

26) M. Ikebe, T. Aiba, H. Onishi, and S. Watanabe: J. Biochem., 83, 1643-1655 (1978).

27) J. A. Spudich and S. Watt: J. Biol. Chem., 246, 4866-4871 (1971).

28) G. E. Youngburg and M. N. Youngburg: $J$. Lab. Clin. Med, 16, 158-166 (1930).

29) H. Harafuji and Y. Ogawa: J. Biochem., 87, 1305-1312 (1980).

30) K. Weber and M. Osborn: J. Biol. Chem., 224, 4406-4412 (1969).

31) W. T. Perrie and S. V. Perry: Biochem. J., 119, 31-38 (1970).

32) A. G. Gornall, C. S. Bardawill, and M. N. David: J. Biol. Chem., 177, 751-765 (1949).

33) H. Kishimura, T. Ojima, and K. Nishita: Nippon Suisan Gakkaishi, 52, 847-851 (1986).

34) A. Goldberg and W. Lehman: Biochem. J., 171, 413-418 (1978).

35) T. Ojima and K. Nishita: J. Biol. Chem., 261, 16749-16754 (1986).

36) W. Lehman: Biochem. J., 163, 291-296 (1977).

37) H. Ichikawa and K. Nishita: Nippon Suisan Gakkaishi, 53, 85-91 (1987). 